\title{
AdS Waves as Exact Solutions to Quadratic Gravity
}

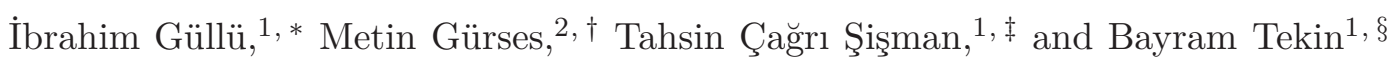 \\ ${ }^{1}$ Department of Physics, \\ Middle East Technical University, 06531 Ankara, Turkey \\ ${ }^{2}$ Department of Mathematics, Faculty of Sciences \\ Bilkent University, 06800 Ankara, Turkey
}

(Dated: March 28, 2011)

\begin{abstract}
We give an exact solution of the quadratic gravity in $D$ dimensions. The solution is a plane-fronted wave metric with a cosmological constant. This metric solves not only the full quadratic gravity field equations but also the linearized ones which include the linearized equations of the recently found critical gravity. A subset of the solutions change the asymptotic structure of the anti-de Sitter space due to their logarithmic behavior.
\end{abstract}

PACS numbers: 04.50.-h, 04.20.Jb, 04.30.-w

\section{Contents}

\section{Introduction}

II. Critical Points of Quadratic Gravities

III. AdS-Wave Metric

IV. Solutions of the Field Equations
A. The $a \neq b$ case
B. The $a=b$ case which includes the critical theory

\section{Conclusions}

VI. Acknowledgments

\section{A. Solution for the Critical Theory with $\xi \neq 0$}

\section{References}

\section{INTRODUCTION}

Quadratic deformations of Einstein's gravity have always attracted attention since the inception of general relativity for various reasons. Initial motivation was to understand the uniqueness of general relativity, but later on it was realized that perturbative quantum gravity required these terms [1]. Generically, quadratic gravity theories at the linearized level describe massless and massive spin-2, and massive spin-0 modes. Massive spin-2 mode ruins perturbative unitarity due to its ghost nature. Recently, a new interest in quadratic theories arose, since it was shown that in three dimensions the ghost disappears [2], and in D-dimensions in the anti-de Sitter (AdS)

\footnotetext{
*Electronic address: e075555@metu.edu.tr

${ }^{\dagger}$ Electronic address: gurses@fen.bilkent.edu.tr

${ }^{\ddagger}$ Electronic address: sisman@metu.edu.tr

${ }^{\S}$ Electronic address: btekin@metu.edu.tr
} 
background, certain quadratic theories become "critical" with only a massless spin-2 excitation just like the Einstein gravity [3, 4]. These observations led us to consider exact solutions to the quadratic gravity models. The existence of a cosmological constant changes the structure of the field equations and the solutions dramatically. For example, the plane wave metric solves all higher order gravity field equations coming from string theory with zero cosmological constant [5, 6]. With a cosmological constant this does not work; however, if one starts with a plane-fronted AdS-wave [7, 8], one may have exact solutions to quadratic curvature gravity models as we show below. In general, save the Schwarzschild-anti-de Sitter solution, no exact solution is known in generic quadratic gravity theories. In some specific theories, such as the Einstein-Gauss-Bonnet theory, some static spherically-symmetric solutions are known [9]; in the new massive gravity of [2], AdSwave solutions were found in [10], and types D and N solutions were found in [11]. In this paper, in generic $D$-dimensions, we find the AdS-wave solutions for general quadratic theories including the critical gravity. By construction, our exact solutions also solve the linearized wave equations.

The layout of the paper is as follows: In Sec. II, we briefly review the quadratic gravity theory and specifically the critical gravity in $D$-dimensions. In Sec. III, we discuss the AdS-wave metric, compute the Riemann and the relevant tensors, and derive the field equations. In Sec. IV, we present the solutions of the field equations deferring one rather cumbersome case to the Appendix.

\section{CRITICAL POINTS OF QUADRATIC GRAVITIES}

This work was inspired by the critical gravity models [3, 4] which are a certain subset of quadratic curvature gravities. Therefore, we will briefly recapitulate these models. The action of the quadratic gravity is

$$
I=\int d^{D} x \sqrt{-g}\left[\frac{1}{\kappa}\left(R-2 \Lambda_{0}\right)+\alpha R^{2}+\beta R_{\mu \nu}^{2}+\gamma\left(R_{\mu \nu \sigma \rho}^{2}-4 R_{\mu \nu}^{2}+R^{2}\right)\right] .
$$

The (source-free) field equations were given in [12, 13] as

$$
\begin{aligned}
& \frac{1}{\kappa}\left(R_{\mu \nu}-\frac{1}{2} g_{\mu \nu} R+\Lambda_{0} g_{\mu \nu}\right)+ 2 \alpha R\left(R_{\mu \nu}-\frac{1}{4} g_{\mu \nu} R\right)+(2 \alpha+\beta)\left(g_{\mu \nu} \square-\nabla_{\mu} \nabla_{\nu}\right) R \\
&+2 \gamma\left[R R_{\mu \nu}-2 R_{\mu \sigma \nu \rho} R^{\sigma \rho}+R_{\mu \sigma \rho \tau} R_{\nu}{ }^{\sigma \rho \tau}-2 R_{\mu \sigma} R_{\nu}{ }^{\sigma}-\frac{1}{4} g_{\mu \nu}\left(R_{\tau \lambda \sigma \rho}^{2}-4 R_{\sigma \rho}^{2}+R^{2}\right)\right] \\
&+\beta \square\left(R_{\mu \nu}-\frac{1}{2} g_{\mu \nu} R\right)+2 \beta\left(R_{\mu \sigma \nu \rho}-\frac{1}{4} g_{\mu \nu} R_{\sigma \rho}\right) R^{\sigma \rho}=0 .
\end{aligned}
$$

The two AdS vacua of the theory satisfy

$$
\frac{\Lambda-\Lambda_{0}}{2 \kappa}+f \Lambda^{2}=0, \quad f \equiv(D \alpha+\beta) \frac{(D-4)}{(D-2)^{2}}+\gamma \frac{(D-3)(D-4)}{(D-1)(D-2)} .
$$

Around any of these vacua, the linearized equations read [12]

$$
c \mathcal{G}_{\mu \nu}^{L}+(2 \alpha+\beta)\left(\bar{g}_{\mu \nu} \bar{\square}-\bar{\nabla}_{\mu} \bar{\nabla}_{\nu}+\frac{2 \Lambda}{D-2} \bar{g}_{\mu \nu}\right) R^{L}+\beta\left(\bar{\square} \mathcal{G}_{\mu \nu}^{L}-\frac{2 \Lambda}{D-1} \bar{g}_{\mu \nu} R^{L}\right)=0
$$

for the metric perturbation $h_{\mu \nu} \equiv g_{\mu \nu}-\bar{g}_{\mu \nu}$. Here, $\mathcal{G}_{\mu \nu}^{L}$ is the linearized Einstein tensor, and $c$ is given by

$$
c \equiv \frac{1}{\kappa}+\frac{4 \Lambda D}{D-2} \alpha+\frac{4 \Lambda}{D-1} \beta+\frac{4 \Lambda(D-3)(D-4)}{(D-1)(D-2)} \gamma .
$$


The critical theory is obtained as follows: One chooses $4 \alpha(D-1)+D \beta=0$, which then kills the massive spin-0 mode, and sets $R^{L}=-\frac{2 \Lambda}{D-2} h=0$. Then, in the transverse gauge $\nabla^{\mu} h_{\mu \nu}=0$, the linearized equations simplify to

$$
(\beta \bar{\square}+c) \mathcal{G}_{\mu \nu}^{L}=0,
$$

or more explicitly

$$
\left(\bar{\square}-\frac{4 \Lambda}{(D-1)(D-2)}-M^{2}\right)\left(\bar{\square}-\frac{4 \Lambda}{(D-1)(D-2)}\right) h_{\mu \nu}=0,
$$

where

$$
M^{2}=-\frac{1}{\beta}\left(c+\frac{4 \Lambda \beta}{(D-1)(D-2)}\right),
$$

and the point $M^{2}=0$ defines the critical point where one is left only with a massless spin-2 excitation.

\section{AdS-WAVE METRIC}

The quadratic field equations are highly nontrivial, therefore the form of the metric ansatz is important in finding solutions. Here, we take the $D$-dimensional AdS-wave metric (which is conformally related to the pp-wave metric) to be in the Kerr-Schild form [14, 15] as

$$
g_{\mu \nu}=\bar{g}_{\mu \nu}+2 V \lambda_{\mu} \lambda_{\nu},
$$

where $\bar{g}_{\mu \nu}$ is the metric of the AdS space. The vector $\lambda^{\mu}=g^{\mu \nu} \lambda_{\nu}=\bar{g}^{\mu \nu} \lambda_{\nu}$ is assumed to be null and geodesic with respect to both $\bar{g}_{\mu \nu}$ and $g_{\mu \nu}$, that is [16]

$$
\lambda_{\mu} \lambda^{\mu}=g^{\mu \nu} \lambda_{\mu} \lambda_{\nu}=\bar{g}^{\mu \nu} \lambda_{\mu} \lambda_{\nu}=0, \quad \lambda^{\mu} \nabla_{\mu} \lambda^{\nu}=\lambda^{\mu} \bar{\nabla}_{\mu} \lambda^{\nu}=0 .
$$

The inverse metric can be found as

$$
g^{\mu \nu}=\bar{g}^{\mu \nu}-2 V \lambda^{\mu} \lambda^{\nu}
$$

which is an exact form. Here, note the similarity with a perturbation analysis where the metric perturbation is defined as $h_{\mu \nu} \equiv g_{\mu \nu}-\bar{g}_{\mu \nu}$; and, at the linearized level, the inverse metric becomes $g^{\mu \nu}=\bar{g}^{\mu \nu}-h^{\mu \nu}$. Now, let us choose the coordinates on AdS to be in the conformally flat form

$$
\bar{g}_{\mu \nu}=\phi^{-2} \eta_{\mu \nu}
$$

where $\phi=k_{\mu} x^{\mu}, k_{\mu}$ is a constant vector, and we choose the flat space coordinates as $x^{\mu}=$ $\left(u, v, x^{1}, \cdots, x^{n}\right)$ with $n=D-2$. This choice of $\bar{g}_{\mu \nu}$ simplifies the construction. Here, $u$ and $v$ are null coordinates, hence more explicitly

$$
\eta_{\mu \nu} d x^{\mu} d x^{\nu}=2 d u d v+\sum_{m=1}^{n}\left(d x^{m}\right)^{2} .
$$

Then, the vector $k_{\mu}=\left(0,0, k_{1}, \cdots, k_{n}\right)$ is related to the cosmological constant as

$$
\bar{R}_{\mu \nu}=\frac{2 \Lambda}{D-2} \bar{g}_{\mu \nu}, \quad \Lambda=-\frac{(D-1)(D-2)}{2 \ell^{2}},
$$


where $\frac{1}{\ell^{2}} \equiv \sum_{m=1}^{n} k_{m} k_{m}>0$, hence we will be working only with $\Lambda<0$ (note that to conform with the usual notation, we introduced the AdS radius $\ell$ ). With this coordinate choice, $\lambda_{\mu}$ naturally becomes

$$
\lambda_{\mu} d x^{\mu}=d u \Rightarrow \lambda^{\mu} \partial_{\mu}=\phi^{2} \partial_{v} .
$$

The function $V$ is assumed not to depend on $v$, that is

$$
\lambda^{\mu} \partial_{\mu} V=0
$$

This choice is extremely important; since, with it, $h_{\mu \nu} \equiv 2 V \lambda_{\mu} \lambda_{\nu}$ becomes transverse $\nabla_{\mu} h^{\mu \nu}=$ $\bar{\nabla}_{\mu} h^{\mu \nu}=0$ and traceless $h \equiv g^{\mu \nu} h_{\mu \nu}=\bar{g}^{\mu \nu} h_{\mu \nu}=0$. Furthermore, as we show below by explicit calculations, the parts of the curvature tensors which are quadratic in $V$ drop out with this choice. It is also important to realize that if $V=\frac{c(u)}{\phi^{2}}$, then $g_{\mu \nu}$ corresponds to just a coordinate transformed version of $\bar{g}_{\mu \nu}$. This is clear since in this case, one can simply define $(u, \tilde{v})$ in such a way that the two-dimensional subspace metric $c(u) d u^{2}+2 d u d v$ becomes $2 d u d \tilde{v}$. This fact will play a role in deciding how our solutions should decay at infinity.

We are now ready to compute the Riemann tensor. The connection corresponding to $g_{\mu \nu}$ splits into two parts

$$
\Gamma_{\alpha \beta}^{\mu}=\bar{\Gamma}_{\alpha \beta}^{\mu}+\Omega_{\alpha \beta}^{\mu},
$$

where $\bar{\Gamma}_{\alpha \beta}^{\mu}$ is the Levi-Civita connection corresponding to $\bar{g}_{\mu \nu}$, which reads as

$$
\bar{\Gamma}_{\alpha \beta}^{\mu}=-\frac{1}{\phi}\left(\delta_{\alpha}^{\mu} \partial_{\beta} \phi+\delta_{\beta}^{\mu} \partial_{\alpha} \phi-\bar{g}_{\alpha \beta} \bar{g}^{\mu \nu} \partial_{\nu} \phi\right) .
$$

The nontrivial part of the connection is given as

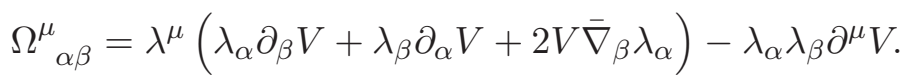

The null vector $\lambda_{\mu}$ with $\partial_{\mu} \lambda_{\nu}=0$ satisfies the following equations, which are frequently used in the computations:

$$
\bar{\nabla}_{\nu} \lambda_{\mu}=\frac{1}{\phi}\left(\lambda_{\mu} \partial_{\nu} \phi+\lambda_{\nu} \partial_{\mu} \phi\right), \quad \bar{\square} \lambda_{\mu}=\frac{1-D}{\ell^{2}} \lambda_{\mu}, \quad \bar{g}^{\mu \nu}\left(\bar{\nabla}_{\mu} \lambda_{\alpha}\right)\left(\bar{\nabla}_{\nu} \lambda_{\beta}\right)=\frac{1}{\ell^{2}} \lambda_{\alpha} \lambda_{\beta},
$$

where $\bar{\square} \equiv \bar{g}^{\mu \nu} \bar{\nabla}_{\mu} \bar{\nabla}_{\nu}$. The Riemann tensor reduces to

$$
R_{\alpha \beta \gamma}^{\nu}=\bar{R}_{\alpha \beta \gamma}^{\nu}+\bar{\nabla}_{\beta} \Omega_{\gamma \alpha}^{\nu}-\bar{\nabla}_{\gamma} \Omega_{\beta \alpha}^{\nu} .
$$

Note that the $\left(\Omega_{\beta \sigma}^{\nu} \Omega_{\gamma \alpha}^{\sigma}-\Omega_{\gamma \sigma}^{\nu} \Omega_{\beta \alpha}^{\sigma}\right)$ part of the Riemann tensor becomes zero, since the explicit calculation of $\Omega_{\beta \sigma}^{\nu} \Omega_{\gamma \alpha}^{\sigma}$ yields a symmetric tensor in $\beta$ and $\gamma$ indices

$$
\Omega_{\beta \sigma}^{\nu} \Omega_{\gamma \alpha}^{\sigma}=-\lambda_{\beta} \lambda_{\gamma} \lambda^{\nu} \lambda_{\alpha} \partial_{\sigma} V\left(\partial^{\sigma} V+\frac{2 V}{\phi} \partial^{\sigma} \phi\right) .
$$

After a lengthy computation, one finds the Riemann tensor as

$$
\begin{aligned}
R_{\alpha \beta \gamma}^{\nu}= & \bar{R}_{\alpha \beta \gamma}^{\nu}-\frac{2 \lambda^{\nu}}{\phi^{2}} \lambda_{[\beta} \partial_{\gamma]} \partial_{\alpha}\left(V \phi^{2}\right)+\frac{2 \lambda^{\nu}}{\phi^{3}} \lambda_{[\beta} \bar{g}_{\gamma] \alpha} \partial_{\rho} \phi \partial^{\rho}\left(V \phi^{2}\right)+\frac{2 \lambda_{\alpha}}{\phi^{3}} \lambda_{[\beta} \partial_{\gamma]}\left(V \phi^{2}\right) \partial^{\nu} \phi \\
& +2 \lambda_{\alpha} \lambda_{[\beta}\left(\partial_{\gamma]} \partial^{\nu} V+\frac{1}{\phi} \partial_{\gamma]} V \partial^{\nu} \phi-\frac{1}{\phi} \delta_{\gamma]}^{\nu} \partial_{\rho} \phi \partial^{\rho} V\right),
\end{aligned}
$$


where as usual $2 A_{[\mu} B_{\nu]} \equiv A_{\mu} B_{\nu}-A_{\nu} B_{\mu}$. Then, the Ricci tensor $R_{\mu \nu} \equiv R_{\mu \rho \nu}^{\rho}$ and the curvature scalar can be computed as

$$
R_{\mu \nu}=\frac{2 \Lambda}{D-2} g_{\mu \nu}-H \lambda_{\mu} \lambda_{\nu}, \quad R=\frac{2 D \Lambda}{D-2},
$$

where $H$ is defined as

$$
H \equiv \frac{4}{\phi} \partial_{\rho} V \partial^{\rho} \phi+\bar{\square} V+\frac{4 V}{\phi^{2}} \partial_{\rho} \phi \partial^{\rho} \phi+\frac{4 \Lambda}{D-2} V .
$$

The following two relations will be used in the field equations

$$
\begin{aligned}
\square R_{\mu \nu} & =-\left(\bar{\square} H+\frac{4-2 D}{\ell^{2}} H+\frac{4}{\phi} \partial_{\rho} H \partial^{\rho} \phi\right) \lambda_{\mu} \lambda_{\nu}, \\
\square\left(V \lambda_{\mu} \lambda_{\nu}\right) & =\left(\bar{\square} V+\frac{4-2 D}{\ell^{2}} V+\frac{4}{\phi} \partial_{\rho} V \partial^{\rho} \phi\right) \lambda_{\mu} \lambda_{\nu} .
\end{aligned}
$$

With our metric ansatz, the field Eqs. (2) split into two parts in the form $A g_{\mu \nu}+B \lambda_{\mu} \lambda_{\nu}$. Trace of this equation yields a relation between the effective cosmological constant and the parameters of the theory exactly given as (3) , where we have used $R=\frac{2 D \Lambda}{D-2}$ and $R_{\tau \lambda \sigma \rho}^{2}-4 R_{\sigma \rho}^{2}+R^{2}=\frac{4 \Lambda^{2} D(D-3)}{(D-1)(D-2)}$. Observe that the $\lambda_{\mu} \lambda_{\nu}$ part does not contribute to the trace equation. To obtain the rest of the field equations, the nontrivial computation is the contraction of two Riemann tensors, that is the $R_{\mu \sigma \rho \tau} R_{\nu}^{\sigma \rho \tau}$ term. After a lengthy computation, one obtains

$$
R_{\mu \alpha \beta \gamma} R_{\nu}^{\alpha \beta \gamma}=\frac{8 \Lambda}{(D-1)(D-2)}\left(R_{\mu \nu}-\frac{\Lambda}{D-2} g_{\mu \nu}\right),
$$

and similarly,

$$
R_{\mu \sigma} R_{\nu}^{\sigma}=\frac{4 \Lambda}{D-2}\left(R_{\mu \nu}-\frac{\Lambda}{D-2} g_{\mu \nu}\right), \quad R_{\mu \rho \nu \sigma} R^{\rho \sigma}=\frac{2 \Lambda}{D-1}\left(R_{\mu \nu}+\frac{2 \Lambda}{(D-2)^{2}} g_{\mu \nu}\right) .
$$

Finally, the remaining field equations become

$$
(\beta \bar{\square}+c)\left(H \lambda_{\mu} \lambda_{\nu}\right)=0,
$$

where $H$ was given in (25). Observe the similarity of this equation to (66). Then, using

$$
\bar{\square}\left(\lambda_{\mu} \lambda_{\nu}\right)=\frac{4 \Lambda}{D-1} \lambda_{\mu} \lambda_{\nu}, \quad \bar{\nabla}_{\rho}\left(\lambda_{\mu} \lambda_{\nu}\right)=\frac{1}{\phi}\left(2 \lambda_{\mu} \lambda_{\nu} \partial_{\rho} \phi+\lambda_{\rho} \lambda_{\nu} \partial_{\mu} \phi+\lambda_{\rho} \lambda_{\mu} \partial_{\nu} \phi\right),
$$

we get

$$
\lambda_{\mu} \lambda_{\nu} \beta\left(\bar{\square}+\frac{4}{\phi} \partial^{\sigma} \phi \partial_{\sigma}+\frac{4(D-3) \Lambda}{(D-1)(D-2)}-M^{2}\right)\left(\bar{\square}+\frac{4}{\phi} \partial^{\rho} \phi \partial_{\rho}+\frac{4(D-3) \Lambda}{(D-1)(D-2)}\right) V=0,
$$

where $M^{2}$ is defined as (8). Therefore, the exact equations of the quadratic gravity reduces to a linear fourth-order wave equation. One can show that putting $h_{\mu \nu}=2 V \lambda_{\mu} \lambda_{\nu}$ in (44) yields (32). When $M^{2}=0$, the theory reduces to the linearized equations of the critical theory of [3, 4]. There is a fine point here: In the critical theory, to get rid off the massive scalar mode, one imposes a relation between $\alpha$ and $\beta, 4 \alpha(D-1)+D \beta=0$; but here these parameters are arbitrary, since $R_{L} \equiv R-\bar{R}$ vanishes identically for the AdS-wave metric. In the next section, we discuss the solutions of (32) in detail. 


\section{SOLUTIONS OF THE FIELD EQUATIONS}

The Eq. (32) is of the form

$$
\left(\bar{\square}+\frac{4}{\phi} \partial^{\sigma} \phi \partial_{\sigma}+b\right)\left(\bar{\square}+\frac{4}{\phi} \partial^{\rho} \phi \partial_{\rho}+a\right) V=0,
$$

with $a=\frac{4(D-3) \Lambda}{(D-1)(D-2)}$ and $b=a-M^{2}$, which are obviously equal for $M^{2}=0$. Whether $M^{2}=0$ or not changes the behavior of solutions dramatically, therefore we will discuss these cases separately.

\section{A. The $a \neq b$ case}

One can define $V=V_{a}+V_{b}$ in such a way that each part satisfies the corresponding second order equation

$$
\left(\bar{\square}+\frac{4}{\phi} \partial^{\rho} \phi \partial_{\rho}+a\right) V_{a}=0, \quad\left(\bar{\square}+\frac{4}{\phi} \partial^{\rho} \phi \partial_{\rho}+b\right) V_{b}=0 .
$$

Without loss of generality, let us choose only the $n^{\text {th }}$ component of the $k^{\mu}$ vector to be nonvanishing; and define $z=x^{n}$, then $\phi=\frac{z}{\ell}$. With this choice, the quadratic equation reduces to

$$
\left(\bar{\square}+\frac{4}{\phi} \partial^{\rho} \phi \partial_{\rho}+a\right) V_{a}=\left(\frac{z^{2}}{\ell^{2}} \partial^{2}+\frac{6-D}{\ell^{2}} z \partial_{z}+a\right) V_{a}=0
$$

where $\partial^{2} \equiv \eta^{\mu \nu} \partial_{\mu} \partial_{\nu}$. Note that the $V_{b}$ equation is similar. Using the separation of variables technique as $V_{a} \equiv \Psi_{a}\left(u, x^{1}, x^{2}, \cdots, x^{n-1}\right) \Phi_{a}(u, z)$, we can split the quadratic equation into two parts:

$$
\begin{aligned}
\left(\vec{\nabla}^{2}+\chi_{a}\right) \Psi_{a}\left(u, x^{1}, x^{2}, \cdots, x^{n-1}\right) & =0 \\
{\left[z^{2} \frac{d^{2}}{d z^{2}}+(6-D) z \frac{d}{d z}+\left(a \ell^{2}-\chi_{a} z^{2}\right)\right] \Phi_{a}(u, z) } & =0
\end{aligned}
$$

where $\chi_{a}$ is an arbitrary real number at this stage and $\vec{\nabla}^{2} \equiv \sum_{i=1}^{n-1} \frac{\partial^{2}}{\partial\left(x^{i}\right)^{2}}$. Note that the $\frac{\partial^{2}}{\partial u \partial v}$ term does not appear because of the $v$-independence of the solution. On the other hand, the solution will have an arbitrary dependence on $u$. Depending on the boundary conditions, $\chi_{a}$ can be continuous or discrete. Then, a formal solution will be of the form

$$
\begin{aligned}
V_{a}\left(u, x^{1}, x^{2}, \cdots, x^{n-1}, z\right)= & \int d \chi_{a} A\left(\chi_{a}\right) \Psi_{a}\left(u, x^{1}, x^{2}, \cdots, x^{n-1} ; \chi_{a}\right) \Phi_{a}\left(u, z ; \chi_{a}\right) \\
& +\sum_{\chi_{a}} B_{\chi_{a}} \Psi_{a, \chi_{a}}\left(u, x^{1}, x^{2}, \cdots, x^{n-1}\right) \Phi_{a, \chi_{a}}(u, z),
\end{aligned}
$$

where $A\left(\chi_{a}\right)$ and $B_{\chi_{a}}$ are arbitrary functions of $u$. Over the entire $\left(u, v, x^{i}\right)$ flat space, the first equation in (36) does not have bounded solutions when $\chi_{a}<0$. Therefore, we will take $\chi_{a} \equiv \xi_{a}^{2} \geq 0$. Here, the discussion bifurcates whether $\xi_{a}=0$ or not. Concentrating on the continuous case, first we start with $\xi_{a} \neq 0$. Then, the solutions are of the form

$$
\Psi_{a}\left(u, x^{1}, x^{2}, \cdots, x^{n-1}\right)=c_{1}(u) \sin \left(\vec{\xi}_{a} \cdot \vec{r}+c_{2}(u)\right),
$$


where $\vec{\xi}_{a}$ is an arbitrary vector with magnitude $\xi_{a}$, and $\vec{r}=\left(x^{i}\right)$. Now, we come to the second equation in (36) which is in the form of the modified Bessel equation for $D=5$, and can be converted to this form for any other $D$ by the following redefinition:

$$
\Phi_{a}(u, z) \equiv z^{\frac{D-5}{2}} f_{a}(u, z),
$$

which then yields

$$
\left[z^{2} \frac{d^{2}}{d z^{2}}+z \frac{d}{d z}-\left(\nu_{a}^{2}+\xi_{a}^{2} z^{2}\right)\right] f_{a}(u, z)=0,
$$

where $\nu_{a}=\frac{1}{2} \sqrt{(D-5)^{2}-4 a \ell^{2}}$. For generic $D$ and nonvanishing $\xi_{a}$, the solution is given as

$$
\Phi_{a}(u, z)=z^{\frac{D-5}{2}}\left[c_{3}(u) I_{\nu_{a}}\left(z \xi_{a}\right)+c_{4}(u) K_{\nu_{a}}\left(z \xi_{a}\right)\right],
$$

where $I_{\nu}$ and $K_{\nu}$ are the modified Bessel functions of the first and second kind, respectively. $V_{b}$ will have the similar solutions with $\nu_{b}=\frac{1}{2} \sqrt{(D-5)^{2}-4 b \ell^{2}}=\frac{1}{2} \sqrt{(D-1)^{2}+4 \ell^{2} M^{2}}$. As we discussed in Sec. III, we require the solution to go like $\frac{1}{z^{2}}$ at the boundary $z=0$; the modified Bessel functions approach $z \rightarrow 0$ as $I_{\nu}(z) \sim z^{\nu}, K_{\nu} \sim z^{-\nu}$ and $K_{0} \sim-\ln z$. Therefore, we keep both $c_{3}$ and $c_{4}$. It is important to realize that $\nu_{a}$ and $\nu_{b}$ are real. This is automatically satisfied for $\nu_{a}$ since $a=-\frac{2(D-3)}{\ell^{2}}$, and $\nu_{a}= \pm \frac{1}{2}(D-1)$. The reality of $\nu_{b}$ puts a constraint on $M^{2}$ which is

$$
M^{2} \geq-\frac{(D-1)^{2}}{4 \ell^{2}} \text {. }
$$

This bound is exactly equivalent to the Breitenlohner-Freedman (BF) bound on the mass of a scalar excitation in AdS [17]. When the bound is saturated, $\nu_{b}=0$ and logarithmic solutions arise.

For the sake of completeness, let us write the solution

$$
\begin{aligned}
V(u, \vec{r}, z)= & z^{\frac{D-5}{2}}\left[c_{a, 1}(u) I_{\nu_{a}}\left(z \xi_{a}\right)+c_{a, 2}(u) K_{\nu_{a}}\left(z \xi_{a}\right)\right] \sin \left(\overrightarrow{\xi_{a}} \cdot \vec{r}+c_{a, 3}(u)\right) \\
& +z^{\frac{D-5}{2}}\left[c_{b, 1}(u) I_{\nu_{b}}\left(z \xi_{b}\right)+c_{b, 2}(u) K_{\nu_{b}}\left(z \xi_{b}\right)\right] \sin \left(\overrightarrow{\xi_{b}} \cdot \vec{r}+c_{b, 3}(u)\right) .
\end{aligned}
$$

The full solution can be obtained by integrating the first part of (43) with respect to $\vec{\xi}_{a}$, and the second part with respect to $\vec{\xi}_{b}$ as in (37). Here, $c_{a, 1}(u)$ and $c_{a, 2}(u)$ depend on $\vec{\xi}_{a}$, and $c_{b, 1}(u)$ and $c_{b, 2}(u)$ depend on $\vec{\xi}_{b}$. In odd dimensions for $D \geq 5, c_{2, a}$ should vanish, otherwise $V$ is unbounded at the boundary of AdS at $z=0$. (Note that we do not worry about the disconnected component of the boundary, which is just the single point $z=\infty$, since this point just compactifies the boundary of AdS from $\mathbb{R}^{D-1} \rightarrow S^{D-1}$.) For generic values of $M^{2}, \nu_{b}$ is not an integer or an odd integer, therefore in general $c_{2, b}$ must be kept.

Let us now consider the $\xi_{a}=\xi_{b}=0$ case. [The case when only one of these parameters vanish follows from the discussion above and the discussion below.] In this case, $\Psi_{a}\left(u, x^{1}, x^{2}, \cdots, x^{n-1}\right)=$ $c(u)+\vec{q}(u) \cdot \vec{r}$, but we take $\vec{q}(u)=0$ to have a bounded solution at $|\vec{r}| \rightarrow \infty$. The other equation reduces to

$$
\left(z^{2} \frac{d^{2}}{d z^{2}}+z \frac{d}{d z}-\nu_{a}^{2}\right) f_{a}(u, z)=0,
$$

whose solution is $f_{a}(z)=c_{a, 1}(u) z^{\left|\nu_{a}\right|}+c_{a, 1}(u) z^{-\left|\nu_{a}\right|}$. Adding also the solution of the $b$ equation, one gets

$$
V(u, z)=c_{a, 1}(u) z^{D-3}+c_{a, 2}(u) \frac{1}{z^{2}}+z^{\frac{D-5}{2}}\left(c_{b, 1}(u) z^{\left|\nu_{b}\right|}+c_{b, 2}(u) z^{-\left|\nu_{b}\right|}\right)
$$


When $M^{2}>0$, because of the last term the spacetime is not asymptotically AdS as one approaches the boundary $z=0$ : Namely, $V(u, z) \sim c_{b, 2}(u) z^{-(2+\epsilon)}$ where $\epsilon>0$, hence $c_{b, 2}(u)=0$. On the other hand, when $0>M^{2}>-\frac{(D-1)^{2}}{4 \ell^{2}}$, all the terms are allowed. When the BF bound (42) is saturated, one has $\left(\nu_{b}=0\right)$

$$
V(u, z)=c_{a, 1}(u) z^{D-3}+c_{a, 2}(u) \frac{1}{z^{2}}+z^{\frac{D-5}{2}}\left[c_{b, 1}(u)+c_{b, 2}(u) \ln \left(\frac{z}{\ell}\right)\right],
$$

which yields an asymptotically AdS metric.

Up to now, we have implicitly assumed $D>3$, but in fact our expressions are also valid for $D=3$ for which (45) and (46) reduce to the results given in [10].

\section{B. The $a=b$ case which includes the critical theory}

In this case, $a=-\frac{2(D-3)}{\ell^{2}}$ and $V_{a}$, as found above (43), is a solution, but this is not the only solution: One should consider the full quadratic theory;

$$
\left(z^{2} \partial^{2}+(6-D) z \partial_{z}-2(D-3)\right)^{2} V=0
$$

Defining $W \equiv\left(z^{2} \partial^{2}+(6-D) z \partial_{z}-2(D-3)\right) V$; so that, $\left(z^{2} \partial^{2}+(6-D) z \partial_{z}-2(D-3)\right) W=$ 0 , since we know the solution of the latter equation from the above discussion, we can simply consider the nonhomogeneous equation, where $W$ is a source term. The $\xi \neq 0$ case is somewhat cumbersome and not particularly illuminating, therefore we defer it to the Appendix, and here study the $\xi=0$ case. Then, the solution to the quadratic equation is

$$
W(u, z)=c_{1}(u) z^{D-3}+c_{2}(u) z^{-2} .
$$

The nonhomogeneous equation becomes

$$
\left(z^{2} \partial^{2}+(6-D) z \partial_{z}-2(D-3)\right) V=c_{1}(u) z^{D-3}+c_{2}(u) z^{-2},
$$

which after rescaling $V(u, z)=z^{\frac{D-5}{2}} f(u, z)$ can be transformed to

$$
\left(z^{2} \frac{d^{2}}{d z^{2}}+z \frac{d}{d z}-\frac{(D-1)^{2}}{4}\right) f(z)=c_{1}(u) z^{\frac{D-1}{2}}+c_{2}(u) z^{\frac{1-D}{2}} .
$$

The general solution of this equation is

$$
V(u, z)=d_{1}(u) z^{D-3}+\frac{d_{2}(u)}{z^{2}}+\frac{1}{D-1}\left(c_{1}(u) z^{D-3}-\frac{c_{2}(u)}{z^{2}}\right) \ln \left(\frac{z}{\ell}\right),
$$

which was also obtained recently in [18], in the context of $D$-dimensional Log gravity. For generic $D$, the solution is not asymptotically AdS, unless $c_{2}(u)$ vanishes. For $D=3$, this equation again reduces to the corresponding expression given in [10].

\section{CONCLUSIONS}

We have found exact AdS-wave solutions in the generic quadratic gravity theory with a cosmological constant. The metrics we have found also solve the linearized field equations of the same 
theory. When we restrict the quadratic theory by choosing $M^{2}=0$, which boils down to eliminating one of the parameters of the quadratic theory, the solutions we found in this case also solve the critical gravity theory defined recently. Depending on the value of $M^{2}$, asymptotic behavior of the solution changes dramatically. Energy and some other physical properties of our solutions, and their conformal field theory duals need to be investigated. It would also be interesting to take the solutions presented here as background and study the spin-2 fluctuations.

Finally, with adjusted $\Lambda$ and $M^{2}$, the metrics we have found will also solve any higher (cubic or more) curvature gravity models and constitute an example of spacetimes studied in [19]. This can be seen as follows: the linearized version of a generic gravity theory constructed from the contractions of the Riemann tensor around AdS will be exactly of the form (41) which is solved by the AdS-wave metrics obtained above. Since by construction the exact field equations reduce to the linearized equations for these solutions, AdS-wave solves the full nonlinear theory at any order in the curvature.

\section{ACKNOWLEDGMENTS}

M. G. is partially supported by the Scientific and Technological Research Council of Turkey (TÜBİTAK) and Turkish Academy of Sciences (TÜBA). The work of I.G., T.C.S., B.T. is supported by the TÜBİTAK Grant No. 110T339, and METU Grant No. BAP-07-02-2010-00-02.

\section{Appendix A: Solution for the Critical Theory with $\xi \neq 0$}

Let us consider the $a=b$ theory for the $\xi \neq 0$ case. The corresponding fourth-order equation reduces to the quadratic nonhomogeneous equation;

$$
\begin{aligned}
\left(z^{2} \partial^{2}+(6-D) z \partial_{z}-2(D-3)\right) V= & z^{\frac{D-5}{2}}\left[c_{1}(u) I_{\nu}(z \xi)+c_{2}(u) K_{\nu}(z \xi)\right] \\
& \times\left[c_{3}(u) e^{i \vec{\xi} \cdot \vec{r}}+c_{4}(u) e^{-i \vec{\xi} \cdot \vec{r}}\right]
\end{aligned}
$$

where $\nu= \pm \frac{1}{2}(D-1)$, but we shall restrict to the positive $\nu$ case; and instead of the sines and cosines we choose the exponentials. This equation can be solved with the help of the Green's function technique. First, we would like to take care of the $\vec{r}$ dependence using the Fourier transform (for the sake of simplicity, here we choose $\xi$ to be continuous, but the discrete case follows similarly)

$$
V(u, z, \vec{r})=\frac{1}{(2 \pi)^{\frac{D-3}{2}}} \int d^{D-3} p \tilde{V}(u, z, \vec{p}) e^{i \vec{p} \cdot \vec{r}},
$$

then (A1) after defining $\tilde{V}(u, z, \vec{p})=z^{\frac{D-5}{2}} f(u, z, \vec{p})$, reduces to

$$
\begin{aligned}
{\left[z^{2} \frac{d^{2}}{d z^{2}}+z \frac{d}{d z}-\left(\nu^{2}+p^{2} z^{2}\right)\right] f(u, z, \vec{p})=} & (2 \pi)^{\frac{D-3}{2}}\left[c_{1}(u) I_{\nu}(z \xi)+c_{2}(u) K_{\nu}(z \xi)\right] \\
& \times\left[c_{3}(u) \delta(\vec{\xi}-\vec{p})+c_{4}(u) \delta(\vec{\xi}+\vec{p})\right] .
\end{aligned}
$$

From the solutions of the homogeneous part, we can construct the Green's function $(\mathcal{O} G=-1)$ as

$$
G\left(z, z^{\prime} ; p\right)=\frac{1}{z^{\prime}} \begin{cases}I_{\nu}(z p) K_{\nu}\left(z^{\prime} p\right) & 0<z<z^{\prime} \\ I_{\nu}\left(z^{\prime} p\right) K_{\nu}(z p) & z^{\prime}<z<\infty\end{cases}
$$


where we have used the Wronskian $W\left\{I_{\nu}(p z) K_{\nu}(p z)\right\}=-\frac{1}{z}$. Therefore, the solution of (A3) becomes

$$
\begin{aligned}
f(u, z, \vec{p})=[ & \left.d_{1}(u) I_{\nu}(z p)+d_{2}(u) K_{\nu}(z p)\right] \\
+ & (2 \pi)^{\frac{D-3}{2}}\left[c_{3}(u) \delta(\vec{\xi}-\vec{p})+c_{4}(u) \delta(\vec{\xi}+\vec{p})\right] \\
& \times \int_{0}^{\infty} d z^{\prime} G\left(z, z^{\prime} ; p\right)\left[c_{1}(u) I_{\nu}\left(z^{\prime} \xi\right)+c_{2}(u) K_{\nu}\left(z^{\prime} \xi\right)\right] .
\end{aligned}
$$

We can carry out the $p$ integrals using

$$
\int d^{D-3} p f(p) e^{i \vec{p} \cdot \vec{r}}=(2 \pi)^{\frac{D-3}{2}} r^{\frac{5-D}{2}} \int_{0}^{\infty} d p f(p) p^{\frac{D-3}{2}} J_{(D-5) / 2}(p r),
$$

where $J_{n}$ is the Bessel function of the first kind. Since the Fourier transform of $I_{\nu}(z p)$ diverges, we must choose $d_{1}(u)=0$. For $D=4$ and $D=5, d_{2}(u)$ must also be zero, since the integral involving $K_{\nu}(z p)$ diverges. Then, the integral involving $K_{\nu}(z p)$ for $D>5$ gives $z^{-\nu}{ }_{2} \tilde{F}_{1}\left(\frac{\nu}{2}-1, \frac{3 \nu}{2}-1, \nu-1,-\frac{r^{2}}{z^{2}}\right)$ where the second factor is the regularized hypergeometric function. As $z \rightarrow 0$, this expression diverges. Therefore, for all $D$, there is no contribution from the homogeneous part, and $c_{2}(u)$ should vanish in the nonhomogeneous part since that term diverges in the $[0, z]$ integral, yielding finally

$$
\begin{aligned}
V(u, z, \vec{r})= & z^{\frac{D-5}{2}}\left[c_{1}(u) e^{i \vec{\xi} \cdot \vec{r}}+c_{2}(u) e^{-i \vec{\xi} \cdot \vec{r}}\right] \int_{0}^{\infty} d z^{\prime} G\left(z, z^{\prime} ; \xi\right) I_{\nu}\left(z^{\prime} \xi\right) \\
= & z^{\frac{D-5}{2}}\left[c_{1}(u) e^{i \vec{\xi} \cdot \vec{r}}+c_{2}(u) e^{-i \vec{\xi} \cdot \vec{r}}\right] \\
& \times\left[K_{\nu}(z \xi) \int_{0}^{z} \frac{1}{z^{\prime}} I_{\nu}\left(z^{\prime} \xi\right) I_{\nu}\left(z^{\prime} \xi\right) d z^{\prime}+I_{\nu}(z \xi) \int_{z}^{\infty} \frac{1}{z^{\prime}} K_{\nu}\left(z^{\prime} \xi\right) I_{\nu}\left(z^{\prime} \xi\right) d z^{\prime}\right] .
\end{aligned}
$$

This solution is valid for a given $\vec{\xi}$. The general solution can be obtained by integrating this solution over the $(D-3)$-dimensional $\vec{\xi}$-space. Here, $c_{1}(u)$ and $c_{2}(u)$ also depend on $\vec{\xi}$. Using Mathematica, one can find the integrals in terms of the hypergeometric function ${ }_{p} F_{q}(a ; b ; z)$ and the digamma function $\psi$

$$
\begin{aligned}
\int_{0}^{z} d z^{\prime} \frac{1}{z^{\prime}} I_{\nu}\left(z^{\prime} \xi\right) I_{\nu}\left(z^{\prime} \xi\right)= & \frac{(\xi z)^{2 \nu}}{2^{2 \nu+1} \nu[\Gamma(\nu+1)]^{2}}{ }_{2} F_{3}\left(\nu, \nu+\frac{1}{2} ; \nu+1, \nu+1,2 \nu+1 ; \xi^{2} z^{2}\right) \\
\int_{z}^{\infty} \frac{1}{z^{\prime}} K_{\nu}\left(z^{\prime} \xi\right) I_{\nu}\left(z^{\prime} \xi\right) d z^{\prime}= & \frac{\xi^{2} z^{2}}{8 \nu\left(\nu^{2}-1\right)}{ }_{3} F_{4}\left(1,1, \frac{3}{2} ; 2,2,2-\nu, \nu+2 ; \xi^{2} z^{2}\right) \\
& -\frac{\Gamma(-\nu)(\xi z)^{2 \nu}}{4^{\nu+1} \nu \Gamma(\nu+1)}{ }_{2} F_{3}\left(\nu, \nu+\frac{1}{2} ; \nu+1, \nu+1,2 \nu+1 ; \xi^{2} z^{2}\right) \\
& -\frac{1}{2 \nu}\left[\ln \left(\frac{z \xi}{2}\right)-\psi(\nu)-\frac{1}{2 \nu}\right]
\end{aligned}
$$

Specifically, for $D=4$, that is $\nu=\frac{3}{2}$, around $z=0$, one has

$$
V(u, z, \vec{r}) \sim\left[c_{1}(u) e^{i \vec{\xi} \cdot \vec{r}}+c_{2}(u) e^{-i \vec{\xi} \cdot \vec{r}}\right] \xi^{3 / 2} z[\ln (\xi z)-1.3963],
$$

which gives an asymptotically AdS metric. 
[1] K. S. Stelle, Phys. Rev. D16, 953 (1977); Gen. Rel. Grav. 9, 353 (1978).

[2] E. A. Bergshoeff, O. Hohm and P. K. Townsend, Phys. Rev. D 79, 124042 (2009).

[3] H. Lu and C. N. Pope, "Critical Gravity in Four Dimensions," arXiv:1101.1971 [hep-th].

[4] S. Deser, H. Liu, H. Lu, C. N. Pope, T. C. Sisman and B. Tekin, Phys. Rev. D 83, 061502 (2011).

[5] R. Güven, Phys. Lett. B 191, 275 (1987).

[6] S. Deser, J. Phys. A 8, 1972 (1975).

[7] M. Gürses, J. Phys. A 14, 1957 (1981).

[8] P. Baekler and M. Gürses, Lett. Math. Phys. 14, 185 (1987).

[9] D. G. Boulware and S. Deser, Phys. Rev. Lett. 55, 2656 (1985).

[10] E. Ayon-Beato, G. Giribet and M. Hassaine, JHEP 0905, 029 (2009).

[11] H. Ahmedov and A. N. Aliev, Phys. Lett. B 694, 143 (2010); Phys. Rev. Lett. 106, 021301 (2011).

[12] S. Deser and B. Tekin, Phys. Rev. Lett. 89, 101101 (2002); Phys. Rev. D 67, 084009 (2003).

[13] I. Gullu and B. Tekin, Phys. Rev. D 80, 064033 (2009).

[14] R. P. Kerr and A. Schild, Proc. Symp. Appl. Math. 17199 (1965); G. C. Debney, R. P. Kerr and A. Schild, J. Math. Phys. 101842 (1969).

[15] M. Gürses and F. Gürsey, J. Math. Phys. 162385 (1975).

[16] T. Dereli and M. Gürses, Phys. Lett. B171, 209-211 (1986).

[17] P. Breitenlohner and D. Z. Freedman, Phys. Lett. B 115, 197 (1982).

[18] M. Alishahiha and R. Fareghbal, "D-Dimensional Log Gravity," arXiv:1101.5891 [hep-th].

[19] A. A. Coley, G. W. Gibbons, S. Hervik and C. N. Pope, Class. Quant. Grav. 25, 145017 (2008). 